\title{
LES IDIOTISMES DANS LA GRAMMAIRE GÉNÉRATIVE
}

\begin{abstract}
Nkollo Mikolaj, Les idiotismes dans la grammaire gênêrative [Idioms within Generative Grammar], Studia Romanica Posnaniensa, Adam Mickiewicz University Press, Poznan,, vol. XXVII: 2001, pp. 103-116. ISBN 83-232-1039-X, ISSN 0137-2475.

The paper deals with the main inadequacies of the generative approach to the analysis of idioms. The apparatus implemented by Chomsky (deep structures, selectional restrictions and kernel sentences) is unable to forsee and explain the irregularities concerning these expressions. The idioms are divided into sub-groups in order to show that their various kinds are to be described in different ways. Some suggestions on how to deal adequately with them are brought about. Although several problems (inflectional morphology, inability to undergo certain transformations) remain unresolved, the proposed improvements afford assimilation of idioms within the lexicon.
\end{abstract}

L'analyse des idiotismes n'occupe pas le premier rang dans le champ d'investigation de la grammaire générative. Il semble que le titre de l'article de Chafe (1968:109) est très significatif à cet égard. Peut-être que le peu d'intérêt porté aux idiotismes par la file génératiste est dû au fait qu'elle ne vise pas à élaborer un modèle de performance, mais plutôt à décrire la compétence linguistique. Toutefois, cette thèse est fortement discutable et semble aller à l'encontre des idées avancées par de Saussure. En effet, le débat sur l'appartenance des idiotismes à la langue ou à la parole (dont les concepts chomskyens précités ne sont qu'une reformulation plus ou moins exacte) remonte au linguiste genevois. D'un autre point de vue, dans la mesure où une locution idiomatique combine plusieurs unités lexicales, il nous est loisible de penser aux rapports syntagmatiques, mais, comme cela va se montrer par la suite, la sélection des lexèmes formant les idiotismes n'est pas aléatoire; il faut donc tenir compte aussi des rapports de substitution qui déterminent les occurences potentielles des mots dans les locutions en question. F. de Saussure estime que, en ce qui concerne les syntagmes, un grand nombre d'expressions appartiennent à la langue (produit qui est enregistré passivement par l'individu à mesure que ce dernier se développe). Il est question, en premier lieu, des locutions toutes faites, auxquelles il est impossible d'apporter des modifications quelle que soit leur nature. Elles sont fournies et transmises par la tradition; la parole (acte individuel de volonté et d'intelligence) n'y peut changer que très peu de choses. 
Pour expliquer la nature des idiotismes, il y a lieu de mentionner les critères qui servent à faire la distinction entre leurs différents types. Selon un des critères, l'idiotisme est une construction dont la traduction littérale d'une langue à une autre est impossible parce qu'elle produit un sens absurde. Ce critère est discutable, comme il y a des "universaux idiomatiques» (cf. enterrer la hache de guerre, to bury the hatchet, zakopać topór wojenny). Toutefois, en dépit de l'existence dans certaines langues des unités sémantiquement équivalentes (dont les sens peuvent être mis en rapport avec les sens des structures analogues d'une autre langue), cette situation n'embrasse qu'un nombre restreint d'expressions. Il paraît donc que la définition susmentionnée des idiotismes peut être admise, ne serait-ce que sommairement. Le critère compositionnel montre que la signification d'un idiotisme ne peut pas se déduire de celle de ses élếments constitutifs et de la façon dont ils sont arrangés. Autrement dit, le sens d'une expression idiomatique n'est pas un simple amalgame du sémantisme de ses parties. Cette façon de traiter le problème est très répandue parmi les linguistes; p. ex. Chafe (ibid.:111) essaie de fusionner le critère compositionnel et l'optique générative. Pour lui, la signification d'un idiotisme, résultant de l'opération de la composante sémantique au niveau de la structure profonde, n'est pas une simple fonction des sens des éléments présents dans cette structure. Il est possible d'objecter à cette approche qu'elle considère l'ensemble des idiotismes d'une langue comme acquis et ne permet pas de décider si nous avons affaire à ce type d'expressions ou à des formations lexicales plus ou moins figées. C'est aussi le critère compositionnel qui se trouve à la base de la définition du phénomène proposée par Katz et Nagel (1974:330). Un idiotisme, selon eux, doit être syntaxiquement complexe et sa valeur sémantique ne résulte pas d'une simple addition des significations de ses éléments et de leur organisation syntaxique. L'originalité de cette conception tient au fait que la structure syntaxique d'une expression idiomatique est, selon Katz et Nagel, nécessairement complexe. C'est une thèse controversable - que faire alors avec hay espagnol dont la structure n'est nullement complexe? C'est pourtant une construction propre à la langue espagnole et dont la traduction, par une forme analogue, dans une autre langue est pratiquement impossible. Si le mot hay ne peut jouer, à lui seul, le même rôle qu'une phrase entière, ce n'est pas le cas avec les mots-phrases populaires Ecrase!, Chapeau! qui ne se laissent pas analyser en unités significatives plus petites, mais dont l'énonciation peut avoir la valeur d'une phrase complète.

Lors de la discussion sur les traits qui opposent les idiotismes à d'autres locutions, j'aimerais évoquer l'opinion de Fraser dont le mérite est d'avoir attiré l'attention sur une caractéristique importante des expressions idiomatiques. Plusieurs d'entre elles sont souvent bien formées au niveau superficiel, mais des problèmes surgissent dès que l'on cherche à établir l'inventaire des transformations qu'elles peuvent subir (cf. Fraser, 1970:23 et 32). Considérons, à titre d'exemple, les idiotismes ayant la forme adjectif-nom ou nom-adjectif ( $\mathrm{p}$. ex. vieille peau, tête carrée). Il faut tenir compte du fait que, selon les prescriptions des génératistes, les constructions de ce type sont dérivées d'une structure sous-jacente reformulable 
comme peau est vieille, tête est carrée ${ }^{\prime}$. Cependant, la transformation en question est de peu d'utilité ici parce qu'elle détruit le sémantisme de l'idiotisme correspondant. De même, la construction relative (peau qui est vieille, tête qui est carrée) ne possède que le sens littéral. Le défaut transformationnel suivant des structures en question est que leur nominalisation n'est pas possible non plus (vieillesse de la peau, carrure de la tête). Les propositions concernant l'analyse de ce type d'idiotismes dans le cadre génératif seront présentées par la suite.

Une autre caractéristique qui semble, d'ailleurs, être la cause des déficiences transformationnelles des expressions idiomatiques consiste dans un fort figement de leur contenu sémantique. Les termes d'un syntagme idiomatique sont ou tendent à être inanalysables du point de vue de l'usage linguistique quotidien. C'est ainsi que mon oeil! n'est plus senti comme un ensemble de deux unités, alors que chacun des termes de cette construction est susceptible de figurer dans une multitude de constructions (mon oeil me fait mal comprise). Il est probable que plusieurs expressions idiomatiques évoluent aussi dans cette direction. Ce trait n'est pas mentionné expressément dans l'étude que Weinreich a consacrée aux idiotismes comme un critère constitutif de ceux-ci, mais il est implicitement inscrit dans la description qu'il fait de la nature du phénomène. Weinreich exprime cette pensée à l'occasion de l'analyse de l'expression shoot the breeze (1969:56) dont les parties ne peuvent pas être remplacées, selon lui, même pas leurs synonymes. Pour illustrer son raisonnement à l'aide d'un exemple français, considérons l'expression taper sur le système. Le remplacement des éléments lexicaux de cette expression par leurs synonymes aboutit à la déformation du contenu sémantique. Cogner sur l'ensemble (qui est une reformulation plus ou moins exacte de l'idiotisme) ne veut plus dire «exaspérer quelqu'un, l'énerver, lui détraquer les nerfs $»^{2}$. Il s'avère, donc, que la paraphrase du sens littéral d'un idiotisme ne reflète pas sa signification.

Une caractéristique apparentée à celle qui vient d'être mentionnée est que souvent l'ordre des éléments lexicaux d'une expression idiomatique ne peut pas être inverti si l'on veut préserver son sens et sa bonne formation. En effet, certains idiotismes sont tellement figés que toute opération de ce type aurait vite fait d'entamer leur sémantisme. Il semble que cette caractéristique se rapporte

${ }^{1}$ „Thus (the adjectival transformation) converts the boy is tall into the tall boy, etc. It is not difficult to show, that this transformation simplifies the grammar considerably, and that it must go in this, not the opposite direction" (Chomsky, op. cit.:72). Cependant, dans les langues où il existe une catégorie sémantico-formelle distincte d'adjectif, ce dernier est, avant tout, une épithète et, secondairement, il est un prédicat, ce qui est confirmé par des recherches historiques (cf. Kurylowicz, 1969:23 et passim). Chomsky semble s'être conforté dans cette conviction malencontreuse après la lecture de la Grammaire générale et raisonnée.

${ }^{2}$ Il y a, toutefois, lieu de noter la possibilité d'échanger les termes dans c'est du chinois et c'est de l'hëbreu, ce qui rappelle la situation avec les variantes libres dans la description phonologique. En effet, l'individu qui se sert des expressions en question n'est nullement obligé de choisir l'une d'entre elles et de rejeter l'autre pour préserver le contenu informationnel et sémantique de son propos. Ce fait est d'autant plus étonnant que l'on n'a pas affaire aux synonymes. Visiblement, le degré de complexité assigné à ces deux langues dans la conscience populaire suffit à les placer au même niveau et à en faire des variantes paradigmatiques qui n'affectent pas la signification. 
particulièrement à ce type d'idiotismes qui, chez Weinreich (ibid.:50), sont appelés «binômes». Il est question d'expressions comme (faire) la pluie et le beau temps, (faire) des pieds et des mains, au fur et à mesure (que), bel et bien, d'ores et déjà, (en raconter) des vertes et des pas mûres, (employer) le fer et le feu, etc. La différence entre ce type d'expressions et celles qui sont reliées à l'aide d'une particule de liaison, mais qui ne sont pas qualifiées d'idiomatiques, consiste dans la possibilité d'échanger la conjonction dans les constructions «normales», tout en préservant le sens (cf. soit boire soit manger, boire ou manger), alors qu'il est impossible de le faire avec des «binômes» (cf. Il y a à boire et à manger dans cette affaire, mais non Il y a à boire ainsi qu'à manger dans cette affaire, Il y a tantôt à boire tantôt à manger dans cette affaire, etc.). L'autre trait inhérent à ces expressions est que l'inversion de leurs termes constitutifs est impossible. Bien que Weinreich constate que la possibilité de telles modifications varie sensiblement en fonction du type d'expressions et de leur enracinement dans la langue, ses remarques concernent la langue anglaise. L'irréversibilité des idiotismes en français est due, à ce qu'il paraît, à des facteurs les plus divers. Si d'ores et déjà et par monts et par vaux paraissent consacrés par la tradition (le changement est inacceptable pour des raisons phonétiques), le figement de employer le fer et le feu relève des facteurs étymologiques et l'ordre des termes de cette expression n'est pas sans conséquences pour le sens ${ }^{3}$. La grammaire transformationnelle étant une théorie essentiellement synchronique, elle n'est pas capable de rendre compte de ce type de phénomènes. Les remarques qui précèdent se rapportent également aux expressions comme: être tout feu tout flamme (pour), (courtiser une fllle) en tout bien toute honneur où nous avons affaire à une asyndète par absence de coordination. Cependant, cette particularité ne se laisse pas expliquer au moyen de la transformation, dite réduction conjonctive (Les sonnets de Mallarmé sont longs et [les sonnets de Mallarmé sont] ennuyeux cf. Ruwet, 1973:329) qui a fait fortune auprès des représentants de la sémantique générative, confrontés à la nécessité d'expliquer l'omission d'une partie de la phrase sous l'effet de la présence d'une conjonction. Il faut donc dire que les travaux des linguistes génératifs s'avèrent être peu concluants dans leurs tentatives de percer à jour les causes d'un tel état de choses.

Pour reprendre l'essence des problèmes débattus en termes plus traditionnels, il est possible de dire que les unités lexicales formant les idiotismes ne sont pas permutables (l'ordre des éléments de leur structure interne ne peut pas être inverti).

${ }^{3}$ Cette expression puise son origine dans les procédés médiévaux de torture; lors des interrogatoires, on lacérait la chair d'un suspect avec des tenailles, on imprimait au fer rouge des signes d'infamie sur ses épaules. Si ces démarches étaient infructueuses, on le condamnait au bûcher. Ces cruautés sont décrites ici en vue d'insister sur la succession des procédés qui semble avoir trouvé son reflet dans l'irréversibilité des termes constitutifs de l'expression en question. Il est intéressant de noter qu'une histoire analogue a eu lieu avec l'expression conquérir par le fer et le feu. L'irréversibilité due aux facteurs étymologiques apparaît également dans l'expression Prendre ses cliques et ses claques qui a été forgée à la base de l'onomatopée clic-clac. La modification de l'ordre des termes reviendrait à mettre en question l'origine de la locution. 
Elles ne sont pas, non plus, commutables (le remplacement d'un terme donné par un autre n'est pas possible). Il en résulte qu'aucun changement sur l'axe paradigmatique ni sur l'axe syntagmatique ne peut être correctement appliqué aux locutions idiomatiques. Cette caractéristique n'entraîne pourtant aucune conséquence négative pour un des principes fondamentaux avancés par les tenants de la grammaire générative, à savoir la récursivité des règles grammaticales et les possibilités syntagmatiques inépuisables d'une langue. Dans cette perspective, n'importe quelle phrase actuelle peut être librement allongée. D'un autre côté, on sait que les expressions idiomatiques accusent une nette tendance à n'admettre aucuñe modification de leur contenu lexical ni de leur structure interne; il s'ensuivrait, alors, qu'elles connaissent des restrictions sévères ayant trait aux rapports syntagmatiques qu'elles entretiennent. Pourtant, la propriété des syntagmes ordinaires d'être répétés ad libitum concerne aussi les idiotismes, ce qui leur permet d'ailleurs de figurer dans une conversation courante où ils apparaissent en compagnie des expressions «normales», souvent à l'intérieur d'une même phrase. Une grammaire peut spécifier, grâce à des adjonctions ou à des enchâssements successifs, un ensemble infini de phrases, même inédites, et elle peut combiner, au sein de ces ensembles, des phrases ordinaires et des expressions idiomatiques. Ces dernières peuvent aussi bien jouer le rôle des propositions principales que des propositions enchâssées (cf. respectivement Elle en a raconté des vertes et des pas mûres, ce qui n'a impressionné personne et Quand elle a commencé à en raconter des vertes et des pas mûres, tout le monde a commencé à applaudir). La récursivité des idiotismes est limitée, tout au plus, par l'impossibilité d'insérer de nouveaux formants lexicaux et grammaticaux dans leur structure et ceci permet de les séparer des expressions ordinaires à ce niveau d'analyse.

Comme il en a déjà été question, le figement des idiotismes se prononce à travers leur résistance à l'égard des transformations. Ceci fait s'interroger sur la rectitude d'un des dogmes présents dans toute la grammaire générative, à savoir - la nature des transformations lexicales, susceptibles de caractériser chaque lexème (ou, à proprement parler, ses traits phonologiques, sémantiques et syntaxiques). Ces transformations inhérentes aux propriétés grammaticales d'un mot $M$ convertissent, selon les chercheurs génératistes, un indicateur syntagmatique initial dépourvu de ce mot en un autre indicateur qui ne diffère de son homologue initial que par la présence de $M$ dans sa structure. Cependant, l'insertion lexicale ne peut pas se pratiquer au hasard quand on a affaire aux locutions figées, car, sauf des exceptions peu nombreuses ${ }^{4}$, elle aurait pour effet la déformation de la correction et de la valeur sémantique de ces locutions (bien qu'elles puissent garder leurs caractéristiques syntaxiques et phonologiques intactes). Ceci montre à quel point les trois caractéristiques grammaticales des unités lexicales dépendent l'une des autres dans les expressions figées. Ce trait des idiotismes semble encore invalider l'utilité

${ }^{4}$ On peut, p. ex., faire aboutir l'expression une fine mouche à la forme une très fine mouche. Dans ce cas-là, son indicateur syntagmatique contiendra, en effet, le nouvel élément. Aussi, est-il possible de préciser les idiotismes verbaux en y insérant les adverbes de manière ou leurs équivalents. 
d'un autre concept tellement chéri par la linguistique générative - les restrictions sélectives. Ces limitations (qu'elles soient nécessités ou prohibitions) concernent les occurences, en positions successives, des unités qui précèdent ou qui suivent. Pour mettre en place une séquence bien formée, il faut que les traits intrinsèques des termes de celle-ci ne s'excluent pas les uns les autres. Comme la notion de traits contextuels ne se rapporte selon Chomsky (1965:155-156) qu'à des verbes et à des adjectifs, il faut que les noms auxquels ils se joignent soient dotés des traits sémantiques formant un tout cohérent avec la signification de ces éléments. C'est ainsi que coasser a le trait (je simplifie): «exige que le sujet désigne une grenouille, un crapaud ou autre nom renvoyant à cette classe de batraciens». Cependant, les expressions idiomatiques échappent à ces limitations, et ceci de deux façons. D'un côté, elles les transgressent (cf. hurler avec les loups, dit à propos d'un humain, alors que le verbe exige que son sujet soit un loup, un chien, etc.), sans en être anomales. De l'autre, il n'y a souvent qu'un seul nom qui puisse être combiné à un verbe figurant dans l'idiotisme, même s'il y en a d'autres qui possèdent les mềmes traits sémantiques et lui sont substituables dans des conditions normales (cf. avoir d'autres chats à fouetter et non pas tigres, léopards ou n'importe quel autre félin, sans mentionner les autres mammifères). Ces caractéristiques renforcent encore le statut non-commutable des idiotismes; en même temps qu'elles leur interdisent de subir les transformations.

Comme il va en être question, ni les transformations qui intéressent une phrase (p. ex. la passivation), ni celles qui en embrassent deux (transformations par coordination ou par subordination) ne peuvent changer que rarement la structure des idiotismes. Ceci tient au fait que leurs termes constitutifs deviennent indissociables, alors qu'ils sont libres ailleurs (c.à.d. dans des expressions non-idiomatiques). Les conséquences dues à cet état de choses consistent dans la perte du sens propre des éléments lexicaux formant une locution idiomatique et le fonctionnement autonome d'une telle expression qui acquiert, dès son figement, un sens complet. La caractéristique d'un idiotisme tient, alors, à ce qu'il n'est pas susceptible d'être analysé au moyen de certains outils élaborés par la grammaire transformationnelle. Il s'avère que le recours à la structure profonde ne permet nullement de saisir la signification de certaines expressions idiomatiques. Normalement, l'expression comme j'ai acheté du lait et des oeufs est dérivée d'une structure sous-jacente ayant, approximativement, la forme: «je acheter [passé] [dét] lait + je acheter [passé] [dét] oeuf [pl]». Cependant, il serait inadéquat d'étudier le sémantisme de employer le fer et le feu comme résultant de la combinaison des significations des unités lexicales constitutives et de leur ordre. Cette remarque se rapporte aussi à plusieurs autres types d'idiotismes. Il faut souligner, néanmoins, que la reconstruction de la structure profonde de certaines locutions idiomatiques n'est pas impossible.

Pour les besoins du présent travail, j'appliquerai deux critères de distinction entre les différents types d'idiotismes, à savoir: la correction (ou non) syntaxique et la possession (ou non) de l'équivalent littéral. L'entrecroisement de ces critêres 
permet de dégager quatre types d'expressions idiomatiques dont un paraît être hautement discutable:

(i) celles qui sont syntaxiquement bien formées et possèdent au moins une interprétation littérale ${ }^{5}$ (p. ex. avaler des couleuvres). C'est ce groupe encore qui paraît embrasser les idiotismes qui, en dépit de leurs structures sémantique et syntaxique un peu irrégulières, ne contreviennent pas aux contraintes imposées par la grammaire et le sens (aussi anomal fût-il). C'est ainsi que Il pleut des hallebardes, construction à sujet discontinu, peut être interprétée par quelqu'un qui ne connaît pas son sens à l'avance, comme «il pleut, mais avec une telle intensité que les gouttes ressemblent aux hallebardes». Il est intéressant de noter, à ce propos, qu'on ne dit pas Il neige des hallebardes, ce qui témoigne du degré auquel certaines locutions sont figées.

(ii) celles qui sont correctes du point de vue syntaxique, mais dont l'interprétation littérale pose des problèmes (p. ex. avoir des larmes dans la voix);

(iii) celles qui contreviennent à des règles syntaxiques et qui ne possèdent pas, en même temps, d'interprétation littérale (p. ex. n'en pouvoir mais, s'embrasser à bouche que veux-tu).

(iv) l'existence d'une quatrième classe d'idiotismes - ceux qui sont syntaxiquement incorrects, mais qui possèdent un équivalent littéral - reste une question à éclairer. Même si ce type n'est pas lacunaire et s'il existe dans la langue des expressions mal-formées auxquelles il soit possible d'attribuer au moins une lecture, la situation n'est pas fréquente. Une des candidates capables de saturer cette classification semble être l'expression être cucul la praline. La transgression syntaxique est due à la place de l'article; en effet le français impose aux séquences de ce type l'ordre article - adjectif - nom. Les difficultés interprétatives liées à être cucul la praline seront discutées par la suite.

Il convient, tout d'abord, d'analyser les différences entre les expressions idiomatiques syntaxiquement correctes dont les unes peuvent, par exemple, revêtir la forme avoir des larmes dans la voix et les autres sonner les cloches à quelqu'un. Ces deux types different entre eux. Il est difficile, voire impossible, d'assigner une interprétation littérale à la première expression, tandis la seconde, elle, possède un homologue littéral, notamment - faire en sorte que les cloches rendent un son destiné à être entendu par quelqu'un. La deuxième construction est sémantiquement correcte. La cause pour laquelle l'expression avoir des larmes dans la voix (ou être suspendu aux lèvres de quelqu'un) ne peut pas être comprise mot à mot est qu'elle contient une combinaison anomale (bizarre, étrange) de concepts sémantiques constitutifs.

${ }^{5}$ On pourrait distinguer ici d'autres types d'idiotismes, p. ex. ceux qui sont syntaxiquement bien formés, mais qui possèdent plus d'une interprétation littérale (cf. tomber sur un bec qui peut être interprété comme: 1) trouver par hasard un bec, 2) tomber de sorte que le bec le premier fasse contact avec la terre). Mais, comme l'objet de ce travail est l'analyse des idiotismes et non pas leur classification, une telle tâche paraît surérogatoire. 
L'analyse des idiotismes dans l'optique générative exige qu'on reconstruise leur structure profonde. Comme il en sera question par la suite, c'est, entre autres, à ce niveau que les différents types d'expressions idiomatiques different entre eux. Avant de passer à une analyse plus détaillée de ce problème, la réflexion sur les particularités des structures profondes qui sous-tendent les idiotismes paraît pertinente. Une des possibilités qui se présentent est d'admettre que les structures sous-jacentes aux expressions idiomatiques revêtent une même forme que celle qui a été proposée par Chomsky (ibid.:64-68 et passim) pour l'analyse des constructions «normales». Il semble, toutefois, qu'il faut tenir compte des deux caractéristiques que je vais brièvement aborder. Plusieurs idiotismes présentent des défectuosités transformationnelles, c.à.d. il est impossible de les soumettre à certaines conversions, p. ex. à la passivation (cf. *Son acte de naissance a été avalé par lui), la topicalisation (cf ${ }^{*} C^{\prime}$ est son acte de naissance qu'il a avalé), etc. Le résultat de ces opérations ferait penser plutôt au sens littéral des constructions en question. Deuxièmement, les occurences d'un idiotisme syntaxiquement correct et doué d'un homologue littéral sont plus fréquentes que celles de cet homolgue. Les causes de cet état de choses ne sont cependant pas très claires. Si l'on adopte le point de vue de Chafe (op. cit.:123), qui prétend que la signification d'une expression idiomatique ressemble à celle d'une unité lexicale singulière, on doit se poser la question de savoir si c'est toujours le cas. Il n'y a, à ce qu'il semble, aucune preuve à cela. C'est vrai que avaler son acte de naissance siginifie, tout simplement, mourir, mais il y a toute une série didiotismes dont le sens ne se laisse pas exprimer en un mot (cf. être cucul la praline, être Gros-Jean comme devant). Selon Chafe, les occurences des idiotismes sont plus fréquentes que celles de leurs équivalents littéraux, de même que la fréquence de ' $m$ ' est plus grande que celle de $\mathrm{p}$. ex. la syllabe [me] dont ' $m$ ' n'est qu'un des éléments. Une plus grande fréquence des expressions idiomatiques est due plutôt à des raisons pragmatiques et résulte d'une utilité plus élevée de ces expressions par rapport à celle de leurs homologues littéraux.

On doit donc se poser la question si les idiotismes comme sonner les cloches à quelqu'un possèdent une même structure profonde que leurs homologues littéraux. Selon l'opinion de Lakoff (1976:70-71) la structure superficielle d'une locution idiomatique est identique à la structure superficielle issue de la dérivation d'une expression «régulière» qui, à son tour, doit être partiellement ou complètement bien-formée. Ce point de vue semble pertinent également pour les représentations sémantiques sous-jacentes à des idiotismes. Pourtant, l'identité des structures profondes ne permet de différencier les lectures littérale et idiomatique qu'au moment de l'interprétation sémantique. La composante sémantique, opérant au niveau profond, doit apporter l'information sur la différence entre les deux lectures. Elles se distinguent, entre autres, par les relations téléologiques (pour la lecture littérale: faire entrendre le son des cloches; pour la lecture figurée: p. ex. faire changer le comportement de quelqu'un), instrumentales (pour la lecture mot à mot: en frappant avec un marteau ou en tirant les cordes; pour la lecture figurée: en 
employant des paroles acerbes), etc. Il s'ensuit que l'attribution à deux types de lectures des structures profondes distinctes semble être justifiée. Mais, il y a aussi d'autres raisons à faire cela. Considérons, à titre d'exemple, l'expression avaler son acte de naissance. La structure profonde de la lecture mot à mot de cette locution doit faire cas du rapport de possession. Par contre, au sens figuré, avaler son acte de naissance ne contiendra pas cette relation thématique, car ceci produirait une déformation de son contenu sémantique.

Un autre problème s'esquisse à l'occasion de l'analyse des idiotismes qui ne possèdent pas d'homologues littéraux. Les difficultés surgissent aussi bien sur le plan syntaxique que sémantique. Pour reprendre l'idée de Chafe (op. cit.:114), si les idiotismes comme être cucul la praline ou n'en pouvoir mais figuraient dans les séquences engendrées par la composante syntaxique, cette composante serait empiriquement inadéquate (le résultat de son application - la structure de surface pourrait contenir des constructions aberrantes). On doit se demander si cette déficience transformationnelle écarte la possibilité de reconstruire la structure profonde convenable. Jusqu'ici l'élaboration d'une telle structure n'a pas été effectuée. Par contre, la structure profonde des locutions idiomatiques dotées d'équivalents mot à mot peut être reconstituée. Bien qu'il reste à éclairer si cette structure est identique pour les lectures figurée et littérale, cette observation montre que les possibilités explicatives d'un des concepts génératifs les plus importants sont restreintes.

Pourtant, les idiotismes dépourvus de correspondant littéral ne sont pas les seuls à échapper à la possibilité de reconstruire leur structure profonde. Il en est ainsi, car ce défaut peut tenir à d'autres facteurs que ceux dont il vient d'être question. Il s'avère, en outre, que les expressions parfaitement traduisibles en langage ordinaire peuvent, elles aussi, être atteintes de ce problème. Dans une première étape de la grammaire générative (cf. Chomsky, 1957:45), la phrase de base (censée représenter l'organisation syntaxique profonde) se compose d'un noyau et d'un autre constituant (appelé modalité) qui représente les éléments obligatoires dont la présence déclenche une transformation appropriée et décrit de la sorte le statut modal de l'expression superficielle. C'est ainsi que la phrase Mes parents m'ont sonné les cloches pendant une heure sera définie comme Déclarative (élément modal obligatoire), Affirmative et Active (éléments facultatifs). Par contre, la question Quelle mouche le pique? serait, dans cette optique, Interrogative et Active. Le deuxième élément constitutif de la structure profonde est le noyau qui se compose d'une phrase nominale suivie d'une phrase verbale. Mais, il est en même temps stipulé que le noyau représente une phrase active, déclarative et affirmative (c.à.d. la phrase nucléaire). La conséquence en est que, initialement, toutes les phrases susceptibles d'apparaître en structure superficielle sont des phrases nucléaires et ce n'est que grâce à l'action des transformations déclenchées par la modalité qu'elles peuvent devenir interrogatives, exclamatives ou impératives (pour ne citer que les éléments modaux obligatoires). Cette hypothèse ne pose aucun problème analytique avec sonner les cloches à quelqu'un, mais la situation prend un 
mauvais tour dès lors qu'il faut reconstruire la forme profonde d'un idiotisme qui ne fonctionne qu'en guise d'une question (directe ou indirecte). Si l'on s'en tient rigoureusement aux conséquences qu'entraîne la construction bipartite de la structure profonde, telle qu'elle vient d'être décrite, il faut admettre que la phrase nucléaire, sous-jacente à Quelle mouche le pique? revêt la forme Une mouche le pique. C'est à cette étape que viennent s'ajouter les transformations effectuées par la composante modale qui métamorphosent Une mouche le pique en une question spécifique. On n'a désormais besoin que d'une insertion lexicale tenue de placer l'adjectif interrogatif quelle à la place appropriée. Un tel point de vue ne tient aucun compte du fonctionnement réel des expressions idiomatiques; la preuve en est que Quelle mouche le pique?, au sens de "pourquoi se met-il en colère», ne peut subir aucune transformation modale et qu'il est possible, tout au plus, de l'enchâsser dans une phrase matrice ${ }^{6}$.

Il convient de signaler les problèmes liés au sémantisme des expressions syntaxiquement incorrectes. Ils résultent des autres facteurs que ceci a eu lieu avec des idiotismes syntaxiquement bien formés. Nous avons ici affaire à la transgression des règles de sous-catégorisation stricte dont le but est de diviser une catégorie lexicale donnée en sous-catégories distinctes en fonction des contextes catégoriels où elle apparaît. Cette défectuosité se rapporte aussi bien aux locutions qui possèdent une interprétation sémantique possible qu'à celles qui en sont dépourvues. Les règles de sous-catégorisation stricte introduisent dans un symbole complexe les traits déterminés par le contexte catégoriel de la suite où figure un morphème donné. Pour que l'insertion d'un lexème dans la position indiquée par le symbole complexe soit réalisée, il faut que les règles en question remplissent les exigences imposées à l'ensemble des traits catégoriels de ce lexème par les informations inscrites dans ce symbole. Sinon, le remplacement du symbole complexe par une unité (lexicale ou grammaticale) donnée ne pourra pas avoir lieu. C'est ce type de règles qui n'est pas respecté par la structure syntaxique de n'en pouvoir mais et par les expressions similaires (s'embrasser à bouche que veux-tu). La conjonction qui y figure se combine soit avec deux propositions, soit avec deux termes appartenant à une mềme partie du discours. Ce n'est qu'en leur compagnie qu'elle apporte une information susceptible d'être comprise par les interlocuteurs. Cependant, mais, à elle seule, n'est pas informative; elle ne l'est pas non plus en compagnie d'une seule proposition (*il est allé, mais, *mais, elle se cassera la jambe), ni d'un seul nom (*un vieillard, mais) ou d'un seul adjectif (*mais affable). Autrement dit, la règle $\mathrm{CONJ} \rightarrow \mathrm{SC} /\{\mathrm{P}, \mathrm{NP}, \mathrm{Adj} \ldots\} \ldots\{\mathrm{P}, \mathrm{NP}, \mathrm{Adj} \ldots\}$ analyse la catégorie CONJ en un symbole complexe qui contiendra les traits de sous-catégorisation différents, suivant que CONJ apparaît entre deux propositions, deux

${ }^{6}$ Cette remarque concerne aussi les idiotismes, tels que: Ecrase! ou Vogue la galère! où l'on a affaire à des modalités impérative et exclamative, respectivement. Il est impossible de reconstituer leurs structures profondes, car il serait très naif de penser qu'elles peuvent être dérivées des phrases déclaratives. La reconstruction de leur noyau est, d'ailleurs, d'autant plus difficile qu'elles ne se composent pas d'une phrase nominale suivie d'une phrase verbale. 
noms, deux adjectifs, etc. Seul le respect de cette règle assure l'intelligibilité de l'énoncé comportant mais. Toutefois, les règles de sous-catégorisation stricte s'avèrent être un outil conceptuel dont l'utilité lors de l'analyse sémantique est insuffisante. La grammaire générative laisse, une fois de plus, sans réponse le problème de l'informativité des expressions comme n'en pouvoir mais, qui, en dépit de leur structure interne aberrante, transmettent un message compréhensible pour des personnes parlant français.

L'aspect sémantique des idiotismes dépourvus de la lecture mot à mot est un autre point important auquel les instruments analytiques élaborés par la grammaire générative viennent se heurter. Il faut faire attention à ce que le manque des correspondants littéraux des expressions comme être cucul la praline entraîne leur indétermination sémantique. En effet, l'interprétation de la signification des idiotismes en question est loin d'être toujours évidente. La solution de ce problème semble être considérablement facilitée par la notion de trait de transfert, proposée par Weinreich (1972:62-63). Or, l'analyse de être cucul la praline comme "être cucul comme une praline» (ce qui paraît être une interprétation la moins éloignée de la structure syntaxique et la plus probable pour l'individu qui ne connaissait pas son sens avant) semble sémantiquement controversable. Elle transgresse les restrictions sélectives - un objet physique non-animé ne peut pas être décrit comme doué des traits contenus dans le sémantisme de cucul. Ou bien il y a manquement à la règle selon laquelle niaiserie exige un sujet humain, ou bien l'interprétation de praline aboutit à son humanisation. Cette deuxième possibilité, conforme, à ce qu'il semble, à la signification de l'expression, est développée par les traits de transfert. Ce mécanisme exerce son action par contamination, c.à.d. si l'élément lexical doué d'un trait de transfert [+ tf] apparaît en compagnie d'un autre élément, ce dernier se verra attribuer ce trait aussi. Pour appliquer cette conception à l'analyse de l'exemple en question, il faut d'abord reconnaître que le trait [+ Imperfection Morale] est inhérent au sémantisme de cucul. Ceci veut dire que n'importe quelle unité lexicale, à condition qu'elle figure en compagnie de cet adjectif, sera douée du trait [+ Imperfection Morale]. Comme il n'y a que les humains qui peuvent être entachés de ce trait, la procédure aboutit à "personnaliser» le terme précédé de cucul. Toutefois, comme cela va se montrer par la suite, l'utilité des traits de transfert est limitée.

L'analyse des idiotismes, selon les prescriptions des chercheurs génératistes, peut être grandement facilitée si l'on aboutit à les placer dans un dictionnaire (ou lexique) construit selon les règles de Chomsky (cf. particulièrement ses remarques sur les processus flexionnels 1965: 172-177). L'anatomie d'une entrée lexicale chomskyenne se présente comme suit: c'est l'ensemble des matrices de traits phonologiques, syntaxiques et sémantiques. Les transformations lexicales s'effectuent grâce à la règle dont la tâche consiste à substituer les entrées lexicales aux symboles postiches ou complexes dans la séquence pré-terminale si certaines conditions contextuelles (p. ex. la sous-catégorisation stricte) sont remplies. La séquence pré-terminale, engendrée par la composante catégorielle, est une 
succession de formants grammaticaux et de symboles postiches ou complexes (c.à.d. des lieux d'insertion des unités lexicales). Après l'application de la règle en question autant de fois que le nombre de lieux d'insertion dans la séquence pré-terminale l'exige, on obtient une suite terminale. Celle-ci est ensuite sujette à l'interprétation sémantique (dans la terminologie de Katz, 1966:165, il est question de la lecture dérivée). Weinreich (1969:57-59) propose de modifier légèrement cette conception. Il ajoute au dictionnaire une liste d'idiotismes où chaque entrée est une séquence de morphèmes, douée de son indicateur syntagmatique et de la description de sa signification. Chaque adresse contient, en outre, la spécification des traits contextuels (tenus d'indiquer dans quelles conditions les unités lexicales peuvent être insérées dans une séquence donnée de morphèmes).

Le problème se pose alors de décider si un idiotisme donné doit être considéré comme une unité intégrale ou si ce sont ses parties constitutives qu'on doit soumettre à l'analyse. Comme il en sera question par la suite, cette décision possède une importance cruciale pour la description des idiotismes dans l'optique générative, ce qui tient à la nécessité de se prémunir contre les transformations inadmissibles. Considérons d'abord le traitement unitaire de la locution idiomatique défendre son bifteck comme phrase verbale de Le garçon a défendu son bifteck. Il faut d'abord esquisser l'image arborescente représentant la structure syntagmatique de cette phrase. La particularité du traitement unitaire exige que la phrase verbale ne soit pas décomposée en verbe suivi du déterminant de la phrase nominale-complément; la phrase verbale est considérée comme indivisible. La représentation de la forme phonologique du syntagme verbal est le deuxième élément. La caractéristique syntaxique spécifie qu'il est question d'un verbe qui exige un sujet animé dont le nombre peut être singulier ou pluriel et qui, d'autre part, n'accepte pas de phrase nominale (autrement dit, nous avons affaire à un verbe intransitif, ce qui résulte du traitement unitaire) ${ }^{7}$. Enfin, la reformulation de la signification renseigne sur le sens de l'expression, notamment "défendre ses intérêts». Les avantages du traitement unitaire consistent à éviter une insertion prohibée des modificateurs des termes constitutifs (*Le garçon a défendu son petit bifteck). En outre, cette approche interdit la pluralisation de bifteck (*Le garçon a défendu ses biftecks) et sa nominalisation (*La défense de son bifteck par le garçon). Finalement, le traitement unitaire n'admet pas la passivation parce que l'expression est considérée comme un verbe intransitif (*Le bifteck a été défendu par le garçon semble ne refléter que le sens littéral). Mais, le traitement unitaire présente aussi certains défauts importants: la représentation phonologique contient une séquence de sons qui ne se rencontre pas normalement en français - /drs/. Les désavantages morphologiques sont peut-être encore plus flagrants. Il faut, par exemple, s'interroger sur la place des désinences flexionnelles lors de la conjugaison. Si le traitement unitaire doit être

${ }^{7}$ L'importance de ces traits contextuels est, entre autres, qu'ils prohibent l'occurence d'un sujet ayant les mêmes caractéristiques morphologiques ou/et phonétiques que garçon (p.ex. puceron, courçon, etc.) et qui pourraient être en rapport de substituabilité virtuelle avec lui. C'est ainsi que la grammaire générative tient compte de la distinction saussurienne: syntagmatique $v s$ paradigmatique. 
appliqué rigoureusement, les formes suivantes doivent apparaître: *Je défendre mon biftecks, *Tu défendre ton bifteckais, *Il défendre son biftecka (sans mentionner les conjugaisons composées). L'éventuelle nominalisation serait, elle aussi, absurde, p. ex.: * La défendre son bifteckation.

Le traitement d'un idiotisme par le prisme de ses parties constitutives (formant par formant) exige que tous les termes d'une expression donnée soient analysés isolément. Or, la forme phonologique, les restrictions sélectives et les significations littérales des lexèmes constituant la locution en question sont spécifiées. Le sens figuré, supplémentaire dans cette perspective, de défendre son bifteck doit faire partie de son entrée lexicale. Dans ce cas-là, les irrégularités phonologiques disparaissent parce que les frontières des mots sont nettement fixées. La structure sous-jacente peut être de recours lors de l'analyse de l'organisation syntaxique de l'expression, ainsi que de son contenu sémantique (au moins, dans la mesure où le sémantisme de défendre est gardé dans les significations littérale et figurée). Mais, cette approche présente, elle aussi, des défauts, cette fois-ci au niveau sémantique. Les constructions analysées ne se laissent pas paraphraser (protéger son bifteck, défendre son steak n'apportent l'information que sur le sens littéral de l'expression). Pour cette raison il paraît difficile, voire impossible, de reconstruire leurs représentations sémantiques. Il semble que même les traits de transfert, évoqués ci-dessus, ne permettent pas de résoudre le problème. Il s'avère donc que ni le traitement unitaire ni l'analyse des termes constitutifs ne facilitent pas la compréhension de la nature sémantique des expressions idiomatiques.

Pour que les idiotismes puissent être dûment analysés (c.à.d. sans produire des absurdités sémantiques) au moyen des concepts traditionnels de la grammaire générative, il faut apporter à la composante de base quelques modifications structurales. Elles consistent à dresser une liste de locutions idiomatiques et à la placer à l'intérieur de la composante en question. Chacune des expressions idiomatiques y est traitée comme une entrée lexicale distincte, ayant aussi bien le caractère d'un formant grammatical que lexical. Dans la composante catégorielle, tenue d'engendrer les suites pré-terminales (c.à.d. des séquences de formants grammaticaux et de symboles complexes ou postiches $-\Delta$ ), les idiotismes constituent une catégorie majeure, tout comme les noms, les verbes, etc. Indexée de la sorte, la nouvelle catégorie, susceptible, le cas échéant, de jouer le même rôle qu'une phrase, est directement dominée par $S$ (phrase) et domine elle-même ses termes constitutifs. Cette proposition résulte de la volonté d'éviter les transformations inadmissibles. Linsertion lexicale convertit ces séquences en une structure formée par la substitution des entrées lexicales idiomatiques aux occurences des symboles complexes ou postiches appropriés. Les restrictions syntagmatiques que connaissent les idiotismes sont déterminées par les règles de sous-catégorisation (concernant les co-occurences potentielles avec d'autres catégories grammaticales) et de sélection (tenues de préserver la compatibilité sémantique). La liste des locutions idiomatiques doit contenir aussi une règle qui prohibe l'occurence d'un formant lexical aux propriétés catégorielles et sémantiques 
identiques à celles d'un lexême figurant dans l'idiotisme (c.à.d. de son synonyme), mais dont l'insertion à la place du terme approprié aurait «détruit» la signification. C'est pour cette raison que chaque idiotisme doit être considéré comme une entrée lexicale distincte (cf. Jackendoff, 1975:662). L'élément facultatif, venu s'interposer à ce moment-là, est la reformulation du sémantisme de la locution analysée prise intégralement (c.à.d. non formant par formant). Là où l'idiotisme possède un homologue littéral, il est indispensable de décider de quelle signification il est question. Ceci est réalisé au moyen d'un trait contrastif censée prévenir les éventuels malentendus (le trait en question doit certainement faire cas du contexte d'apparition de la locution). Enfin, la composante transformationnelle convertit la suite terminale en une structure superficielle où la composante phonologique (dont la tâche consiste à conférer une interprétation phonétique à des expressions) vient exercer son impact. Une fois ces opérations accomplies, l'indicateur syntagmatique dérivé et la structure de surface informent les participants à la communication qu'ils ont affaire à une phrase (ou structure) grammaticale dont la forme, parfois aberrante, n'affecte nullement l'informativité ni la correction.

\section{BIBLIOGRAPHIE}

Chafe W. (1968), Idiomaticity as an Anomaly in the Chomskyan Paradigm, Foundations of Language

4, D. Reidel Publishing Company, Dodrecht-Boston, pp. 109-127.

Chomsky N. (1957), Syntactic Structures, Mouton, The Hague.

Chomsky N. (1965), Aspects of the Theory of Syntax, MIT Press, Cambridge, Massachusetts.

Fraser B., (1970), Idioms within Transformational Grammar, Foundations of Language 6, D. Reidel Publishing Company, Dodrecht-Boston, pp. 22-42.

Jackendoff R. (1975), Morphological and Semantic Regularities in the Lexicon, Language 51 (3), The Linguistic Society of America, Baltimore, pp. 639-671.

Katz J.J. (1966), The Philosophy of Language, Harper and Row Publishers, New York.

Katz J.J., Nagel R. (1974), Meaning Postulates and Semantic Theory, Foundations of Language 11, D. Reidel Publishing Company, Dodrecht-Boston, pp. 311-340.

Kuryłowicz J. (1969), Kilka uwag o tzw. gramatyce transformacyjno-generatywnej, Biuletyn Polskiego Towarzystwa Językoznawczego XXVII, Zakład Narodowy im. Ossolińskich, Wrocław, pp. 23-27.

Lak off G. (1976), Some Thoughts on Transderivational Constraints (in:) Nawrocka-Fisiak J., ed., pp. 62-71.

Nawrocka-Fis iak J. ed. (1976), Readings in Generative Semantics, Wydawnictwo UAM, Poznań.

Puhvel J. ed. (1969), Substance and Structure of Language, University of California Press, Berkley Los Angeles.

Ruwet N. (1973), An Introduction to Generative Grammar, North-Holland Publishing Company, Amsterdam-London (trad. de Ruwet 1968).

Weinreich U. (1969), Problems in the Analysis of Idioms (in:) Puhvel J., ed., pp. 23-79.

Weinreich U. (1972), Explorations in Semantic Theory, Mouton, The Hague. 